\title{
Coordination of Virtual Inertia Control and Frequency Damping in PV Systems for Optimal Frequency Support
}

\author{
Qiao Peng, Yongheng Yang, Tianqi Liu, and Frede Blaabjerg
}

\begin{abstract}
The photovoltaic (PV) systems are being required to support the grid more flexibly than ever before. One of the emerging demands is the frequency regulation, including the virtual inertia control (VIC) and the frequency damping control (FDC). To achieve the full-range frequency regulation, i.e., to deal with under-frequency and over-frequency issues, power reserve is necessary for PV systems, which should be properly utilized to realize optimal frequency support. In this context, a novel coordination strategy for the VIC and the FDC in PV systems is proposed in this paper. According to relevant grid codes, the key indices to evaluate the frequency quality are identified at first, i.e., the rate of change of frequency $(\mathrm{RoCoF})$, the frequency nadir, and the recovery frequency. Then, the impact of the inertia constant and damping gain on the frequency quality is explored. Accordingly, the coordination strategy of the VIC and the FDC is designed to achieve optimal frequency support with a certain amount of power reserve. Simulations are performed to validate the performance of the proposed control strategy.
\end{abstract}

Index Terms-Frequency damping, frequency stability, photovoltaic (PV) system, power electronic converters, swing equation, virtual inertia control (VIC).

\section{INTRODUCTION}

$\mathrm{T}$ HE global energy revolution is calling for power system reconstruction with more renewable energy. In such a context, the modern power system is becoming dominated by power electronics to integrate renewable energy, which achieves an environmental-friendly power system with considerable possibilities for it to become more flexible and functional [1]. However, the wide application of power electronics introduces stability issues to the power system as well [2]. For instance, with the conventional synchronous generators (SGs) being replaced by distributed power generation systems (DPGSs), it becomes relatively difficult to regulate the grid frequency [3]. In the SG-controlled system, the mechanical

Manuscript received July 29, 2020; revised September 25, 2020; accepted October 18, 2020. Date of publication December 30, 2020; date of current version December 6, 2020. (Corresponding author: Yongheng Yang.)

Q. Peng, Y. Yang, and F. Blaabjerg are with the Department of Energy Technology, Aalborg University, Aalborg 9220, Denmark (e-mail: qpe@et.aau. dk; yoy@et.aau.dk; fbl@et.aau.dk).

T. Liu is with the College of Electrical Engineering, Sichuan University, Chengdu 610065, China (e-mail: tqliu@scu.edu.cn).

Digital Object Identifier 10.24295/CPSSTPEA.2020.00025 inertia and damping of the rotors determine the frequency characteristics [4]. Consequently, the DPGSs, which are generally a combination of renewable energy sources and power converters, should be developed to be active in the grid by providing cost-effective grid support, e.g., the frequency regulation. This becomes mandatory in more and more countries and/or required by international organizations [5]-[7].

The photovoltaic (PV) system, which takes a non-negligible proportion of the worldwide renewable energy [8], is being expected to contribute more to the power system transition. Accordingly, different frequency control schemes for PV systems have been developed. For instance, the DC-link voltage control in the PV systems was modified in [9] to emulate virtual inertia. A synchronous power controller was proposed in [10] to provide virtual inertia from the PV system, which was then adopted for large-scale PV plants [11]. However, these solutions use energy storage units, e.g., DC-link capacitors or additional batteries to realize the inertia emulation, where the power regulation capability of PV systems is not maximized. To facilitate the virtual inertia control (VIC) and frequency damping control (FDC) by regulating the power from PV systems, appropriate measures should be taken toward gridfriendly systems.

Conventionally, PV systems are controlled by a maximum power point tracking (MPPT) algorithm to optimize the energy yield, where the PV systems constantly operate at the maximum power point (MPP). However, with the increasing interest in the flexible power generation as aforementioned, PV systems should be able to regulate the output power more adaptively upon demand, e.g., increasing the PV power when the load increases [12]. To achieve so, reserving a certain amount of power is essential for modern PV systems. Various power reserve control strategies of PV systems were then proposed in the literature, which can be based on incorporating additional sensors [13], master-slave operation of multiple PV units (the MPP was tracked by (a) master PV unit(s) and sent to the slave units) [14], curve-fitting algorithms [15], or periodical MPPT-execution (the real-time MPP was measured by an MPPT algorithm and then the power reserve point could be identified) [16], [17].

Conventionally, the PV systems operating at the MPP can only deal with the over-frequency issues without support from the energy storage units. With the above-reviewed 
power reserve control, it becomes possible for PV systems to provide full-range frequency support in a way to tackle the over-frequency and under-frequency issues. Specifically, the FDC, similar to the primary frequency control of SGs, can be carried out, where the PV power (e.g., the reserved power) is regulated in proportion to the grid frequency deviation [13], [18]. On the other hand, the swing equation can be emulated in the PV power controller to provide virtual inertia [19], [20]. Alternatively, the virtual inertia can be provided by the PV systems with a droop controller, i.e., from the rate of change of frequency (RoCoF) to the PV power reference [21], [22]. However, the tuning of the control gains and the coordination between the frequency controllers have not been sufficiently addressed in the literature, which strongly determines the control performance and further the power system stability with a high penetration of PV systems.

To tackle this issue, the VIC and FDC for virtual synchronous generators (VSGs) and virtual synchronous machines (VSMs) can be benchmarked and referred to [23], [24], where the inertia constant and the damping gain can be selected according to the operation experience from the SGs, or be calculated according to desired transient stability of the active power loop [25]. Moreover, they can also be designed by system-level optimization for improved frequency stability of the entire system [26]. Nevertheless, the frequency control gains were pre-designed to be fixed in these schemes, which may not efficiently utilize the regulation capability of DPGSs. In certain cases, the fixed-parameter design may lose its capability to maintain the system operation.

In fact, different from the SGs, of which the fixed inertia constant and damping gain are naturally determined by the mechanical characteristics of the rotors, the virtual inertia and frequency damping emulated by DPGSs, e.g., the VSGs and PV systems, can be flexibly adjusted. To support the grid frequency more flexibly, the adaptive inertia and frequency damping control of the VSGs was discussed in the literature. For example, the inertia constant of the VSGs can be reduced when the frequency goes back to the initial state during an oscillation [27], which effectively reduces the frequency deviation. Inspired by this, the adaptive damping gain of the VSGs was additionally considered in [28], [29] to further shorten the frequency stabilization settling time. However, the effectiveness of these control methods in steady-state frequency deviation reduction has not been validated. Moreover, the limitation of the available peak power for frequency regulation was not considered for the VSGs, where the energy for regulation was from associated energy storage systems (ESSs) that were usually assumed to have exceptionally large capacities. Other attempts were also made in the literature to tune the VSG control parameters in real-time to reduce the transient power provided by the ESSs while improving the grid frequency dynamic under disturbances. In those cases, optimization methods [30] or advanced artificial intelligence algorithms [31] can be employed. Nevertheless, although the entire energy consumption is effectively reduced for costoriented operation, the available power limitation has been

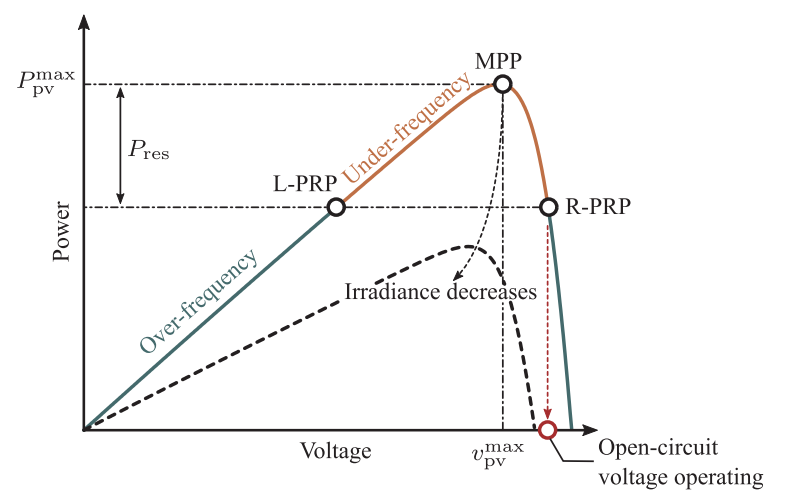

Fig. 1. Illustration of the power reserve operation (power-voltage curves) of PV systems, where $P_{\mathrm{pv}}^{\max }$ and $P_{\text {res }}$ are the maximum PV power and the power reserve, $v_{\mathrm{pv}}^{\max }$ is the $\mathrm{PV}$ voltage corresponding to $P_{\mathrm{pv}}^{\max }(\mathrm{MPP}$ - maximum power point, L-PRP - left power reserve point, R-PRP - right power reserve point).

ignored as well, which is, however, the key to the frequency control of PV systems.

Especially in PV systems, considering that the reserved power is usually unchanged during a certain period, no matter which power reserve control method is applied, the reserved power should be properly coordinated within the VIC and FDC. Only in this way, the power regulation capability of PV systems can be maximized, and optimal and cost-effective frequency support is then achieved. Thus, to improve the grid frequency quality, this paper proposes a novel coordination strategy of the VIC and FDC for PV systems. To highlight the contribution of the proposed control strategy to optimal power reserve utilization, the under-frequency issues are exemplified in this paper. Notably, it is also feasible in overfrequency issues in terms of optimal frequency support. The rest of the paper is organized as follows. The control of PV systems, including the power reserve control, is introduced in Section II to lay the foundation for frequency regulation. The frequency quality requirement from relevant grid codes and the impact of the VIC and FDC on the frequency quality are explored in Section III. Accordingly, the coordination strategy of the VIC and FDC for PV systems with a certain amount of power reserve is proposed in Section IV, and the efficacy of the proposed strategy is validated by simulations in Section V. Finally, the conclusion is given in Section VI.

\section{Control of PV Systems With Power Reserve}

The energy yield from PV systems is highly dependent on the solar irradiance and the ambient temperature, and the power-voltage (P-V) curve of PV panels with different irradiance levels is shown in Fig. 1. As seen in Fig. 1, with the increasing PV voltage, the PV power will increase first and decrease, which generates an inflection point on the P-V curve, referring to as the MPP. Conventionally, the PV system adopts an MPPT algorithm to maximize the power generation. However, with the demand of flexible power support, the power reserve control of PV systems is expected, as shown in Fig. 1. Notably, the maximum PV power, $P_{\mathrm{pv}}^{\max }$, is measured by the power reserve control strategy in real-time [13]-[17], while 


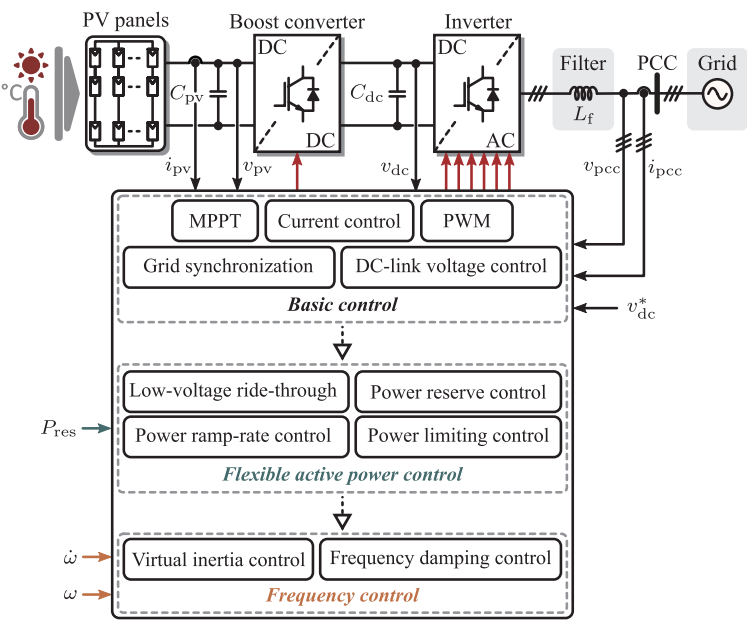

Fig. 2. Control diagram of a two-stage three-phase PV system with frequency regulation, where $\omega$ is the grid angular frequency $(\omega=2 \pi f), C_{\mathrm{pv}}$ and $C_{\mathrm{dc}}$ are the boost capacitor and the DC-link capacitor, $L_{\mathrm{f}}$ is the inverter filter inductance, $i_{\mathrm{pv}}$ and $v_{\mathrm{pv}}$ are the PV current and voltage, $i_{\mathrm{pcc}}$ and $v_{\mathrm{pcc}}$ are the current and voltage of the point of common coupling (PCC), and $v_{\mathrm{dc}}$ and $v_{\mathrm{dc}}^{*}$ are the actual value and reference of the DC-link voltage (MPPT - maximum power point tracking; PWM - pulse width modulation).

the power reserve, $P_{\text {res }}$, is usually set by the system operator. As it is out of the scope of this paper, the details on the power reserve control are not demonstrated. To achieve the power reserve, the PV system should operate at the power reserve point (PRP) instead of the MPP. Due to the non-monotonic P-V relationship, there will be two PRPs, i.e., the left-PRP (L-PRP) and the right-PRP (R-PRP), corresponding to the PRP on the left and right side of the MPP, respectively, as shown in Fig. 1. Although the PV system responds more quickly at the R-PRP, the PV voltage might jump beyond the open-circuit voltage when the irradiance suddenly drops, as illustrated in Fig. 1. This will result in stability issues. Thus, the L-PRP is generally adopted for the power reserve operation of PV systems [32]. Various power reserve control strategies have thus been developed in the literature by controlling the PV systems to operate at the L-RPP [16], [17], [33].

With the reserved power, the full-range frequency control becomes possible for PV systems, where the PV system can increase the output power for under-frequency issues and reduce the output power to tackle over-frequency issues, as shown in Fig. 1. Considering the demand of frequency regulation, the typical control system of a two-stage three-phase PV system is shown in Fig. 2. Generally, the DC-link voltage of the twostage PV system is controlled by grid-connected inverter, while the PV system output power is regulated by boost converter. Furthermore, the grid synchronization is mandatory, which is usually realized by a phase-locked loop (PLL) system. In addition, the dual-loop current control strategy is typically applied to the inverter, of which the reference is generated by the DC-link voltage controller.

As for the boost converter, an MPPT algorithm, a direct PV power control, or a direct PV voltage control can be employed as the basic control to identify the MPP or the PRP. Based on that, various flexible active power control schemes can be
TABLE I

Grid Code for the Northern Europe Grid on the Frequency Quality [34]

\begin{tabular}{lc}
\hline \hline Description & Requirement \\
\hline Rated grid frequency & $50 \mathrm{~Hz}$ \\
Thresholds of normal operation & $\pm 0.1 \mathrm{~Hz}$ \\
Maximum instantaneous frequency deviation & $\pm 1 \mathrm{~Hz}$ \\
Maximum steady-state frequency deviation & $\pm 0.5 \mathrm{~Hz}$ \\
Frequency restoration range & $\pm 0.1 \mathrm{~Hz}$ \\
Time to recover frequenc & Not required \\
Time to restore frequency & $\pm 15 \mathrm{~min}$ \\
RoCoF withstanding capability & \\
& $\pm 2.5 \mathrm{~Hz} / \mathrm{s}$ \\
\hline \hline
\end{tabular}

* Required by local system operators, where the regulation code of the Danish power grid is applied [35].

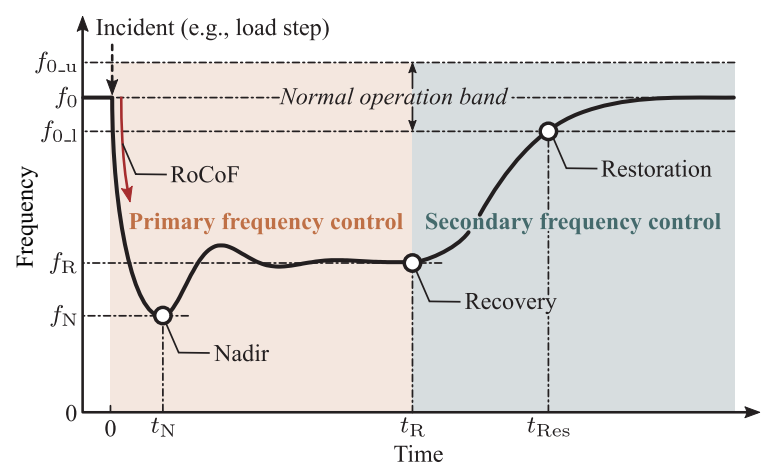

Fig. 3. Frequency dynamics during an under-frequency issue, where $f_{0}$ is the rated grid frequency with $f_{0 u}$ and $f_{01}$ being its upper and lower nominal operation thresholds, respectively, $f_{\mathrm{N}}$ is the frequency nadir, $f_{\mathrm{R}}$ is the recovery steady-state frequency, $t_{\mathrm{N}}$ is the time to reach the frequency nadir, $t_{\mathrm{R}}$ is the time to recover the frequency, and $t_{\text {Res }}$ is the time to restore the frequency (RoCoF rate of change of frequency).

realized, e.g., the power reserve control, the power limiting control, etc. Furthermore, developed from the power reserve control, the frequency control can be achieved, including the VIC and the FDC, as shown in Fig. 2. Notably, as the power reserve is usually invariable, the coordination strategy of the power reserve for different frequency controller should be carefully designed for optimal grid support performance.

\section{GRid Frequency Quality and Regulation}

The frequency is a key parameter in SG-based power systems, which synchronizes the power equipment with the grid. Thus, the requirements of the frequency quality are fundamental in grid codes, which should be properly considered when designing the grid frequency support strategies of PV systems. The frequency quality requirements set by different transmission system operators (TSOs) might vary. For example, the Northern Europe grid code is exemplified in Table I, where it can be seen that not only the steady-state operating range of the frequency is required, but also the frequency transient dynamics are constrained.

As an example, the frequency dynamic in an underfrequency issue is shown in Fig. 3. When the incident happens (e.g., loss of generation), the frequency decreases, which results 


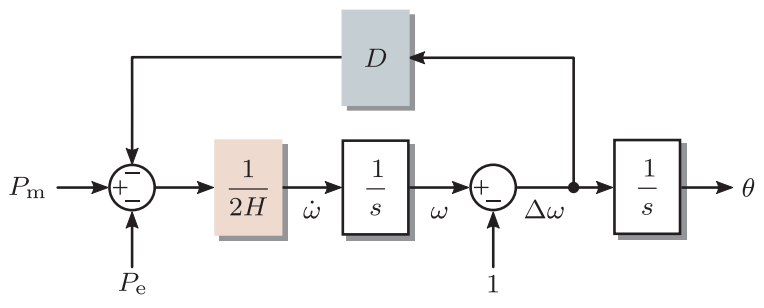

Fig. 4. Diagram of the swing equation of the SG rotors, where $\Delta \omega$ indicates the frequency deviation.

in a relatively large RoCoF (usually measured within a given time window). After the detection of the incident, the primary frequency control is enabled, and the frequency will gradually increase after reaching the nadir. Then, the frequency will oscillate around a new steady-state, indicating that the frequency is recovered, as shown in Fig. 3. However, the steady-state frequency is far from its nominal. Subsequently, the secondary frequency control is activated, where the system control center will re-allocate the output power reference of each power generation unit and then, the frequency starts to increase to the steady-state within the normal operation band. At this point, the frequency is restored. In Fig. 3, several frequency stability indices are highlighted, according to the frequency quality requirements given in Table I. Those include the frequency nadir, $f_{\mathrm{N}}$, the recovery steady-state frequency, $f_{\mathrm{R}}$, the RoCoF, and the time to restore the frequency, $t_{\text {Res }}$. As the primary frequency control can be solely realized by the power generating units, whereas the secondary frequency control is carried out by the control center of the entire system, the frequency stability indices related to the primary frequency control are of interest for the local power generating units. Thus, to design the grid frequency support control of the PV systems, the frequency nadir, the RoCoF, and the recovery frequency should be focused on.

Conventionally, the grid frequency is regulated by the SG rotors, where the mechanical inertia and damping determine the frequency characteristics, following the swing equation. To properly design the VIC and FDC of PV systems, the mechanism of the swing equation should be investigated first. Specifically, the swing equation is given as

$$
\left\{\begin{array}{l}
P_{\mathrm{m}}-P_{\mathrm{e}}=2 H \dot{\omega}+D(\omega-1) \\
\dot{\theta}=\omega-1
\end{array},\right.
$$

where $P_{\mathrm{m}}$ and $P_{\mathrm{e}}$ are the mechanical power and the electromagnetic power of the SG, respectively, $H$ and $D$ are the inertia constant and damping coefficient, $\theta$ is the internal voltage phase angle of the SG, and all variables here and thereafter are per-unit values.

The swing equation can be graphically represented, as shown in Fig. 4, where it can be seen that the inertia constant $H$ and the damping gain $D$ determine the dynamics of the active power and the frequency. Specifically, the inertia constant mainly decides the derivative of the frequency (i.e., the RoCoF), while the damping gain affects more the frequency

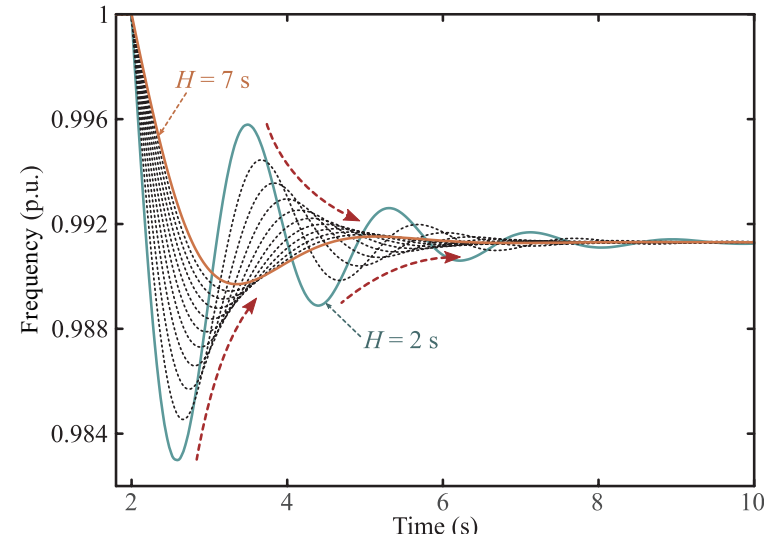

(a)

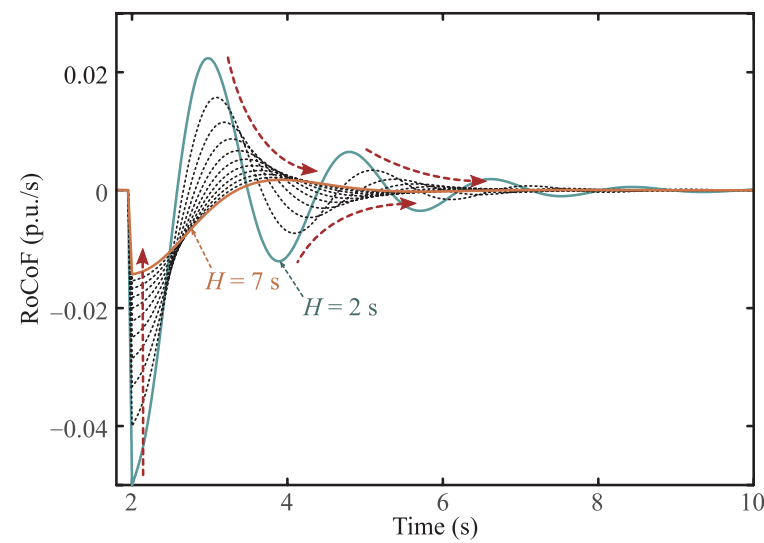

(b)

Fig. 5. Dynamics of the frequency and RoCoF under a load step with increasing inertia constants: (a) grid frequency and (b) RoCoF.

deviation. In turn, in large-scale power systems, the RoCoF is highly dependent on the system total inertia, whereas the steady-state frequency deviation relies more on the system total damping. To further demonstrate the impacts of inertia constant and damping gain on the frequency dynamics, an analysis is conducted as follows.

\section{1) Inertia Constant}

According to (1) and Fig. 4, the responses of the frequency and the RoCoF under a $20 \%$ load step when the inertia constant increases from $2 \mathrm{~s}$ to $7 \mathrm{~s}$ are shown in Fig. 5, where the damping gain is fixed to be 3 p.u. It can be seen in Fig. 5 that the frequency will oscillate for a period before reaching the recovery frequency. When the inertia constant increases, the frequency nadir is significantly increased as well as the time to the nadir. After reaching the nadir, the frequency gradually goes to the rated value until reaches an upper-inflection (where the RoCoF reaches zero). Although the larger inertia makes the system take more time to the upper-inflection, the frequency at this point is dramatically decreased. Finally, after several oscillations, the frequency reaches the recovery point much faster with the large inertia. Notably, the inertia constant does not affect the recovery frequency, as the inertia performs as a buffer to the transient energy, which will fall to zero after reaching the new steady-state, as shown in Fig. 5. As for the 


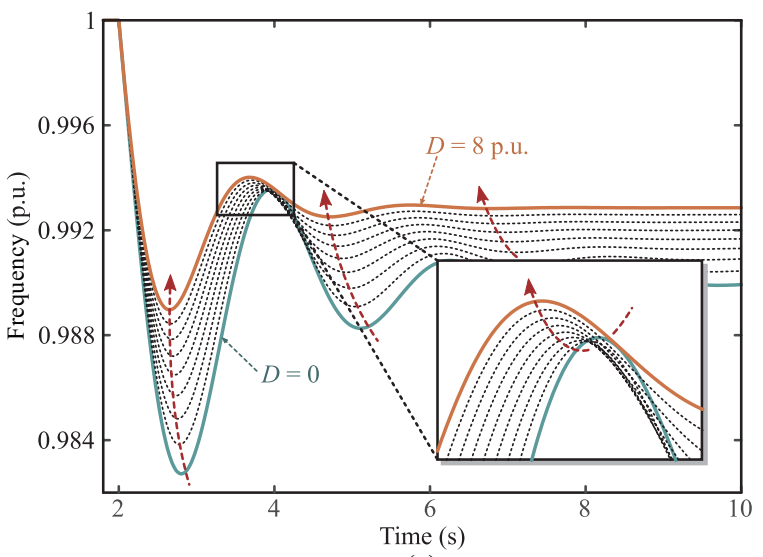

(a)

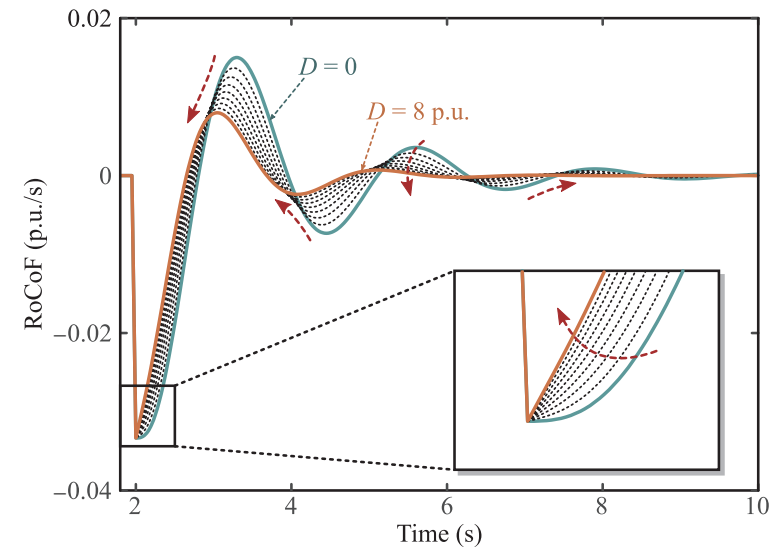

(b)

Fig. 6. Dynamics of the frequency and RoCoF under a load step with increasing damping gains: (a) grid frequency and (b) RoCoF.

RoCoF, it can be observed in Fig. 5 that it is effectively reduced with the increasing inertia. In such a case, several emergencies due to the large RoCoF, e.g., disconnection of the SGs or loss of generation can be avoided. Then, the frequency quality and further the system stability are improved.

\section{2) Damping Gain}

A similar study is conducted while the damping gain increases from 0 to 8 p.u. and the inertia constant is fixed to be $3 \mathrm{~s}$. The analysis results are shown in Fig. 6. It can be seen in Fig. 6 that the frequency nadir is increased with a larger damping gain, whereas the time to the nadir is almost not changed. Notably, the larger damping gain can effectively increase the lower-inflections (smaller under-shoots) in the following oscillations, but the upperinflections are barely affected, as shown in Fig. 6. Finally, the frequency will go to the recovery state more quickly with a larger damping gain, where the recovery frequency is much closer to the rated value. Consequently, part of the regulatory burden for the rest of the power generation units in the system is released. As for the RoCoF, it can be seen in Fig. 6 that the damping gain barely affects the RoCoF before the frequency nadir, where the RoCoF is usually the largest during an oscillation. Although the RoCoF can be reduced by increasing the damping gain after the frequency nadir, it is relatively minor for the frequency quality improvement.

It can be concluded from the above analysis that a large inertia constant can increase the nadir and reduce the RoCoF, while the damping gain contributes more to an increased frequency nadir (a larger nadir) as well as the steady-state frequency deviation. The above summary matches well the analysis in [29].

\section{Coordination of Virtual Inertia Control and Frequency DAmping Control in PV Systems}

Inspired by the swing equation characteristics of the $\mathrm{SG}$ rotors, see (1), the frequency control of PV systems can be realized in two aspects, i.e., the VIC and the FDC, aiming to enhance the inertia and damping of the grid. For an SG, its inertia and damping characteristics are determined by the mechanical property of the rotor, which are unalterable. However, different from the SGs, the PV systems can regulate the output power more flexibly to provide adaptive inertia and damping to the grid. Then, the grid frequency quality can be effectively improved. In this section, a novel frequency control strategy of PV systems is proposed to optimally coordinate the VIC and FDC with a certain amount of power reserve.

\section{A. Virtual Inertia Control and Frequency Damping Control}

As aforementioned, to achieve the VIC, the PV output power should be regulated in proportion to the RoCoF, i.e.,

$$
P_{\mathrm{pv}}^{\mathrm{VIC}}=2 H_{\mathrm{pv}} \dot{\omega}
$$

where $P_{\mathrm{pv}}^{\mathrm{VIC}}$ is an additional PV power reference for the VIC and $H_{\mathrm{pv}}$ is the virtual inertia of the PV system that provides. The virtual inertia of the PV system is limited by the available power for regulation as well as the RoCoF threshold defined by grid codes, as shown in Table I, i.e.

$$
\left\{\begin{array}{l}
H_{\mathrm{pv}}^{\max }=\frac{P_{\mathrm{res}}^{\mathrm{VIC}}}{2 \dot{\omega}_{\mathrm{t}}}, \\
H_{\mathrm{pv}}^{\min }=0
\end{array}\right.
$$

where $H_{\mathrm{pv}}^{\mathrm{max}}$ and $H_{\mathrm{pv}}^{\min }$ are the upper and lower limits of $H_{\mathrm{pv}}, \dot{\omega}_{\mathrm{t}}$ is the RoCoF threshold set by the system operator and $P_{\text {res }}^{\mathrm{vlC}}$ is the power reserve of the PV system with the VIC.

As for the FDC, it can be realized by regulating the PV output power to be proportional to the frequency deviation as

$$
P_{\mathrm{pv}}^{\mathrm{FDC}}=D_{\mathrm{pv}} \Delta \omega,
$$

where $P_{\mathrm{pv}}^{\mathrm{FDC}}$ is an additional PV power reference for FDC and $D_{\mathrm{pv}}$ is the damping provided by the PV system. The damping gain of the PV system is limited by the available power for regulation and the frequency deviation threshold according to the grid code, which can be expressed as

$$
\left\{\begin{array}{l}
D_{\mathrm{pv}}^{\max }=\frac{P_{\mathrm{res}}^{\mathrm{FDC}}}{\Delta \omega_{\mathrm{t}}}, \\
D_{\mathrm{pv}}^{\min }=0
\end{array}\right.
$$


where $D_{\mathrm{pv}}^{\max }$ and $D_{\mathrm{pv}}^{\min }$ are the upper and lower limits of $D_{\mathrm{pv}}, \Delta \omega_{\mathrm{t}}$ is the steady-state frequency deviation threshold set by the system operator and $P_{\text {res }}^{\mathrm{VIC}}$ is the power reserve of the PV system with the FDC.

Notably, since the PV system output power is emulated as the additional electromagnetic power for the frequency governor, i.e., the SG, the control gain from the RoCoF to the PV power reference should be inversely proportional. Thus, the additional power references, i.e., $P_{\mathrm{pv}}^{\mathrm{VIC}}$ and $P_{\mathrm{pv}}^{\mathrm{FDC}}$, should be negative when being added to the PV power controller.

\section{B. Coordination Control With Certain Power Reserve}

According to Table I, the frequency nadir, the RoCoF, and the recovery frequency are the key indices for the frequency quality. Additionally, the RoCoF usually reaches its largest at the early stage of the frequency incident. Thus, for the frequency support control of PV systems, the objectives for an under-frequency issue are to reduce the RoCoF, especially at the early stage of the oscillation, increase the nadir, and maintain the recovery frequency close to the nominal. As discussed in Section III, by increasing the inertia constant, the RoCoF at the early stage of the oscillation can be effectively reduced, while the nadir can be increased as well. Although a large damping gain can also increase the nadir, it has negligible effect on the RoCoF at the early stage of the disturbance. As for the recovery frequency, it is not affected by the inertia constant, but it can be maintained at a higher level with larger damping gains. Thus, to improve the frequency quality, a large inertia constant should be adopted at the early stage of the frequency event, while a large damping gain is desired after the nadir.

However, in the PV systems, the virtual inertia constant and damping gain are limited by the available power for regulation. Thus, to maximize the frequency support capability of PV systems, the power reserve should be properly coordinated according to the above discussed. Specifically, when an abnormal frequency is detected, the frequency regulation is activated. At this point, the power reserve is used to generate the virtual inertia, where the inertia constant is set to be as large as possible, i.e., $H_{\mathrm{pv}}^{\max }$ in (3). Once the frequency nadir is detected, the power reserve is adopted to provide damping to the frequency oscillation, where the damping gain should be larger, i.e., $D_{\mathrm{pv}}^{\max }$ in (5), and the inertia constant is zero at this time. Notably, the change rates of the inertia constant and damping gain after the nadir should be limited. Otherwise, the instantaneous RoCoF would increase drastically due to the sudden drop of the inertia constant, which may deteriorate the frequency quality. However, the change rates should not be very small, which will lead to a long frequency recovery time. Thus, the design of the rate limits should comprehensively consider the grid requirements on RoCoF and frequency recovery period. During the next period after the nadir, it is assumed that the inertia constant will decrease to zero and the damping gain will increase to the maximum within a given transition time, based on which the rate limits can be calculated.
TABLE II

Strategy of the Proposed Control Method

\begin{tabular}{lccc}
\hline \hline State & $\begin{array}{c}\text { Inertia } \\
\text { constant }\end{array}$ & $\begin{array}{c}\text { Damping } \\
\text { gain }\end{array}$ & $\begin{array}{c}\text { Power reserve } \\
\text { allocation }\end{array}$ \\
\hline $\begin{array}{l}\text { Before the nadir } \\
\text { After the nadir }\end{array}$ & $\begin{array}{c}H_{\mathrm{pv}}^{\max } \\
H_{\mathrm{pv}}^{\max } \Rightarrow 0\end{array}$ & $0 \Rightarrow D_{\mathrm{pv}}^{\max }$ & $\begin{array}{c}P_{\mathrm{pv}}^{\mathrm{VIC}}=P_{\text {res }} \\
P_{\mathrm{pv}}^{\mathrm{FDC}}=P_{\text {res }}\end{array}$ \\
\hline \hline
\end{tabular}

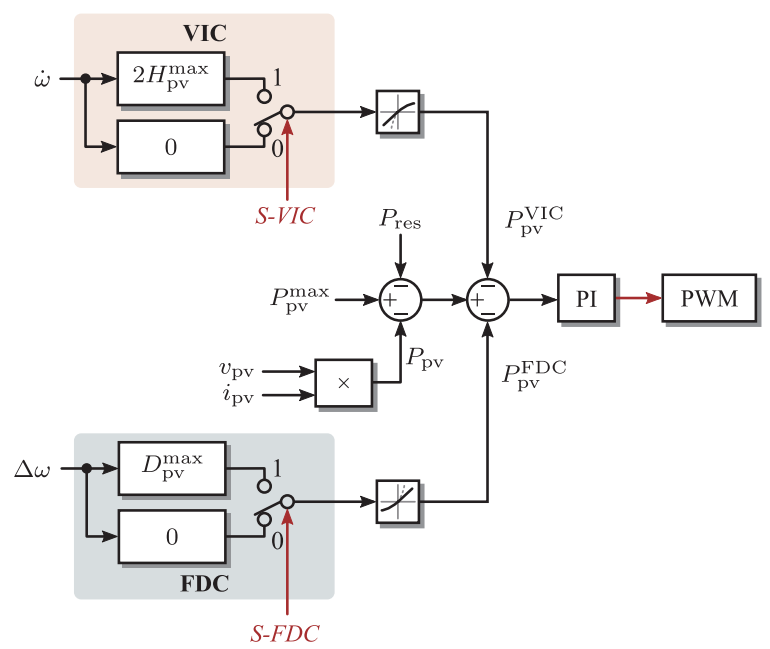

Fig. 7. Diagram of the proposed control method, where $P_{\mathrm{pv}}$ is the instantaneous PV output power, S-VIC and S-FDC denote the switching signals for the VIC and FDC, respectively (PI - proportional integral).

The strategy and diagram of the proposed control method are shown in Table II and Fig. 7, which is implemented in the control of the boost converter (see Fig. 2). Specifically, the PV output power reference is generated by the maximum available power, the power reserve, and the additional reference introduced by the VIC or the FDC. The difference between the power reference and the instantaneous output power will be sent to a proportional-integral (PI) controller, which will generate the duty cycle for the boost converter. It should be mentioned that the calculation (or measurement) of the RoCoF, i.e., $\dot{\omega}$ in Fig. 7, largely affects the performance of the VIC. Improper RoCoF detection may introduce significant noise into the control system, which may further result in system instability. However, the real-time acquisition of the RoCoF is an over-complicated subject that requires much further attention. Thus, to highlight the performance of the proposed frequency regulation strategy, the RoCoF in this paper is directly obtained from the system operator.

Notably, the activation of the VIC and FDC loops is controlled by switching signals, i.e., S-VIC and S-FDC in Fig. 7, which is significantly important for the control strategy. The generation of the control signals is shown in Fig. 8. Three key signals in Fig. 8 are explained as follows.

- Frequency incident detection: It generates the frequency incident signal by an S-R flip-flop. When the frequency moves out of the normal operation band, the signal turns from " 0 " to " 1 ". When the frequency is back to the normal operation band and the RoCoF is zero, the signal returns 


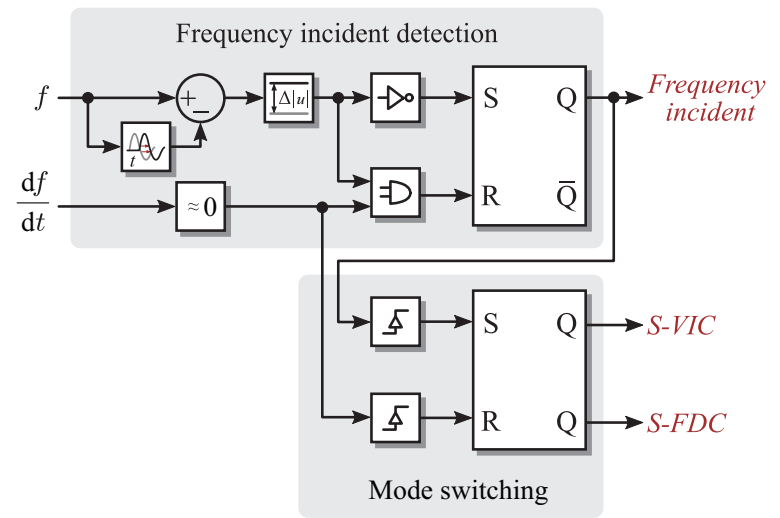

(a)

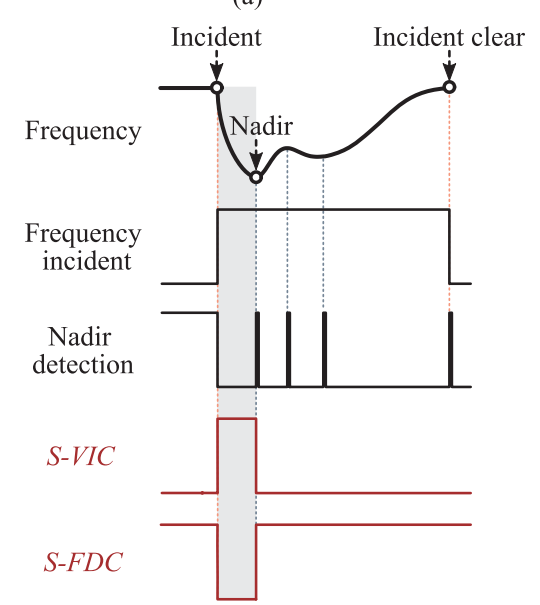

(b)

Fig. 8. Operation logical of the control signals of the VIC and FDC (S-VIC and S-FDC) in Fig. 7: (a) generation of the control signals and (b) example waveform of the signals.

to " 0 ". Then, the PV system waits for the next frequency incident.

- S-VIC: It controls the switching of the VIC by an S-R flip-flop. The rising edge of the frequency incident signal makes the S-VIC signal turn to " 1 " from " 0 ". Then, once the frequency nadir is detected, the signal returns to " 0 ".

- S-FDC: It controls the switching of the FDC. The signal is inverted from the S-VIC signal. Notably, in the steadystate, the S-FDC signal is " 1 ", which makes the PV system operate at the frequency damping mode when waiting for the frequency incidents.

\section{Control Validation}

In this section, simulations are performed on a two-stage three-phase PV system referring to Fig. 2 in MATLAB/Simulink to validate the proposed control method. Furthermore, to clearly clarify the control performance, the grid is islanded with being represented by a small-capacity VSG, of which the control strategy is shown in (1) and the output power is $2 \mathrm{~kW}$ in steady-state. In this case, the real-time RoCoF for frequency regulation is measured in the control system of the VSG. The parameters of the system are given in Table III. A constant solar irradiance level of $1000 \mathrm{~W} / \mathrm{m}^{2}$ and an ambient temperature of
TABLE III

Parameters of the Case-Study System

\begin{tabular}{|c|c|c|}
\hline Parameter & Description & Value \\
\hline \multicolumn{3}{|c|}{ Grid } \\
\hline$P_{\text {rated }}$ & Rated power & $3 \mathrm{~kW}$ \\
\hline$f_{0}$ & Rated grid frequency & $50 \mathrm{~Hz}$ \\
\hline$v_{\text {rated }}^{\mathrm{g}}$ & Rated line-to-line grid voltage & $400 \mathrm{~V}$ \\
\hline \multicolumn{3}{|c|}{ VSG } \\
\hline$P_{\mathrm{VSG}}$ & Steady-state output power & 0.67 p.u. \\
\hline$v_{\mathrm{dc}}^{\mathrm{g}}$ & Rated DC-link voltage & $800 \mathrm{~V}$ \\
\hline$R$ & Speed regulation gain of governor & 0.05 p.u. \\
\hline$T_{\mathrm{G}}$ & Governor time constant & $0.2 \mathrm{~s}$ \\
\hline$T_{\mathrm{T}}$ & Turbine time constant & $0.3 \mathrm{~s}$ \\
\hline$H$ & Inertia constant & $5 \mathrm{~s}$ \\
\hline$D$ & Damping gain & 1 p.u. \\
\hline$L_{\mathrm{f}}^{\mathrm{inv}}$ & Converter-side filter inductance & $4.8 \mathrm{mH}$ \\
\hline$L_{\mathrm{f}}^{\mathrm{g}}$ & Grid-side filter inductance & $2 \mathrm{mH}$ \\
\hline$C_{\mathrm{f}}^{\mathrm{g}}$ & Filter capacitance & $10 \mu \mathrm{F}$ \\
\hline$L_{\mathrm{g}}$ & Grid inductance & $2 \mathrm{mH}$ \\
\hline \multicolumn{3}{|c|}{ PV system } \\
\hline$v_{\mathrm{dc}}^{*}$ & DC-link voltage reference & $650 \mathrm{~V}$ \\
\hline$f_{\mathrm{b}}$ & Boost converter switching frequency & $16 \mathrm{kHz}$ \\
\hline$f_{\text {inv }}$ & Inverter switching frequency & $8 \mathrm{kHz}$ \\
\hline$L_{\mathrm{pv}}$ & Boost converter inductance & $0.5 \mathrm{mH}$ \\
\hline$L_{\mathrm{f}}$ & Inverter filter inductance & $3.5 \mathrm{mH}$ \\
\hline$C_{\mathrm{pv}}$ & Boost converter capacitor & $100 \mu \mathrm{F}$ \\
\hline$C_{\mathrm{dc}}$ & DC-link capacitor & $2800 \mu \mathrm{F}$ \\
\hline$\left(k_{\mathrm{pb}}, k_{\mathrm{ib}}\right)$ & $\begin{array}{l}\text { Boost converter PI controller } \\
\text { parameters, } \mathrm{PI}_{\mathrm{b}}\end{array}$ & $(0.2,20)$ \\
\hline$\left(k_{\mathrm{pdc}}, k_{\mathrm{idc}}\right)$ & $\begin{array}{l}\text { DC-link voltage PI controller } \\
\text { parameters, } \mathrm{PI}_{\mathrm{dc}}\end{array}$ & $(0.2,10)$ \\
\hline
\end{tabular}

$25^{\circ} \mathrm{C}$ are considered in the case study, where the maximum available power of the PV system is $3.04 \mathrm{~kW}$, i.e., $P_{\mathrm{pv}}^{\max }=1.01$ p.u. Considering the power reserve of $0.4 \mathrm{~kW}$, i.e., $P_{\text {res }}=0.13$ p.u., the maximum PV output power to the grid is $2.64 \mathrm{~kW}$.

As shown in Table I, the maximum steady-state frequency deviation and the maximum allowed $\mathrm{RoCoF}$ are $\pm 0.5 \mathrm{~Hz}$ and $\pm 2.5 \mathrm{~Hz} / \mathrm{s}$, respectively. At the early stage of the frequency incident, the power reserve of the PV system is fully utilized to provide virtual inertia. The maximum inertia constant can be calculated according to (3) as

$$
H_{\mathrm{pv}}^{\max }=\frac{0.4 / 3}{2 \times 2.5 / 50}=1.33 \mathrm{~s} .
$$

After the nadir, the power reserve is fully used to provide damping, and the maximum damping gain can be calculated according to (5) as

$$
D_{\mathrm{pv}}^{\max }=\frac{0.4 / 3}{0.5 / 50}=13.33 \text { p.u., }
$$

where the maximum steady-state frequency deviation is considered. With the calculated $H_{\mathrm{pv}}^{\max }$ and $D_{\mathrm{pv}}^{\max }$, the proposed VIC and FDC coordination strategy can be realized according to Table II and Fig. 7. As aforementioned, the design of the change rate limits of the inertia constant and damping gain should consider the grid requirements on the RoCoF and 
frequency recovery time. However, as shown in Table I, the frequency recovery time has not been required by the Danish grid code yet. Thus, the transition time in this case study is designed to be $1 \mathrm{~s}$, referring to the average rolling time window for the RoCoF measurement in practical power systems [36].

Four frequency regulation strategies for PV systems are compared in the following.

\section{1) Fixed Inertia Constant}

The power reserve of the PV system is fully used to achieve the VIC during the entire frequency dynamic response. In this case, the inertia constant $H_{\mathrm{pv}}^{\prime}=H_{\mathrm{pv}}^{\max }=1.33 \mathrm{~s}$, whereas the damping gain is zero.

\section{2) Fixed Damping Gain}

The power reserve of the PV system is utilized to realize the FDC. Notably, no inertia is provided simultaneously to damp the frequency variations. Instead, the frequency nadir, which is limited by the maximum instantaneous frequency deviation, and the peak PV power are solely determined by the damping gain. Thus, the damping gain of this control should be designed from two aspects, i.e., the peak PV power and the PV power at the new steady-state, affecting the maximum instantaneous frequency deviation $( \pm 1 \mathrm{~Hz}$ in Table I) and the maximum steady-state frequency deviation, respectively. The damping gain of this control is calculated as

$$
D_{\mathrm{pv}}^{\prime}=\min \left\{\frac{0.4 / 3}{1 / 50}, \frac{0.4 / 3}{0.5 / 50}\right\}=6.67 \text { p.u., }
$$

while the inertia constant is zero.

\section{3) Fixed Inertia Constant \& Damping Gain}

This control strategy is the most widely applied one in the frequency regulation of PV systems. In this case study, the reserved power is evenly divided to provide virtual inertia and damping, i.e., $0.2 \mathrm{~kW}$ reserved for VIC and FDC, respectively. Notably, the peak PV power for the VIC and the FDC does not appear exactly at the same time [37]. Thus, the peak PV power limitation in designing the inertia constant and damping gain can be considered to be larger than half of the full power reserve. However, the accurate allowance still requires further exploration. In this case study, it is considered that the peak PV power limitation in designing the inertia constant is half of the power reserve, whereas the peak PV power limitation in designing the damping gain is the full power reserve. In such a case, the inertia constant is calculated to be

$$
H_{\mathrm{pv}}^{\prime \prime}=\frac{0.2 / 3}{2 \times 2.5 / 50}=0.67 \mathrm{~s} .
$$

Similar to the fixed damping gain strategy, the damping gain in this strategy is limited by not only the maximum steadystate frequency deviation but also the maximum instantaneous frequency deviation (corresponding to the peak PV power limitation), as the damping gain participates in determining the frequency nadir and peak PV power. Additionally, as mentioned in the above, the peak PV power limitation for designing the damping gain can be considered to be the full
TABLE IV

Strategy of the Alternating Inertia Control Method

\begin{tabular}{cccc}
\hline \hline$\Delta \omega$ & $\dot{\omega}$ & Mode & Inertia constant \\
\hline$>0$ & $>0$ & Accelerating & $H_{\mathrm{pv}}^{\max }$ \\
$>0$ & $<0$ & Decelerating & 0 \\
$<0$ & $<0$ & Accelerating & $H_{\mathrm{pv}}^{\max }$ \\
$<0$ & $>0$ & Decelerating & 0 \\
\hline \hline
\end{tabular}

power reserve. The damping gain is accordingly calculated as

$$
D_{\mathrm{pv}}^{\prime \prime}=\min \left\{\frac{0.4 / 3}{1 / 50}, \frac{0.4 / 3}{0.5 / 50}\right\}=6.67 \text { p.u. }
$$

\section{4) Alternating Inertia Constant}

This control strategy was proposed in [27] for VSGs to improve the frequency dynamics and decrease the energy consumption by tuning the inertia constant. Specifically, the frequency dynamic under disturbances can be divided into two modes, i.e., accelerating mode and decelerating mode, corresponding to the states where the frequency goes beyond and returns to the rated, respectively. In such a case, large inertia can be adopted in the accelerating mode to restrain the frequency deviation, whereas small inertia can be applied in the decelerating mode to make the frequency quickly go back to the rated value. In this case study, the large inertia constant is equivalent to $H_{\mathrm{pv}}^{\max }=1.33 \mathrm{~s}$. The control strategy of this method is given in Table IV.

\section{A. Comparison With the VIC and FDC}

At $t=5 \mathrm{~s}$, a load step of $0.45 \mathrm{~kW}$ (about $10 \%$ ) is applied to the system. The system dynamics are shown in Fig. 9, where the proposed control, the fixed inertia constant control (single VIC), the fixed damping gain control (single FDC) are demonstrated.

According to Fig. 9(a), the performance of different control strategies in terms of frequency control can be summarized as:

- When the frequency control of the PV system is not applied, the frequency drops to $49.28 \mathrm{~Hz}$ and then reaches the recovery frequency as $49.46 \mathrm{~Hz}$ after oscillation. The RoCoF in this case is $-1.10 \mathrm{~Hz} / \mathrm{s}$.

- With the fixed damping gain control, the frequency nadir as well as the recovery frequency are significantly increased, and the RoCoF is slightly reduced.

- In contrast, with the fixed inertia constant control, the RoCoF is considerably reduced. However, although the frequency nadir is also increased, the performance is inferior to the fixed damping gain control. In addition, the recovery frequency is not increased at all, meaning that the PV system does not support the grid frequency after the oscillation.

- Comparably, when the proposed control is adopted, at the early stage of the oscillation, the VIC takes effect and the frequency dynamic is similar to the case with the fixed inertia constant control. Here, the RoCoF is reduced and the nadir is increased. Moreover, the recovery frequency is significantly increased since the PV system is switched 


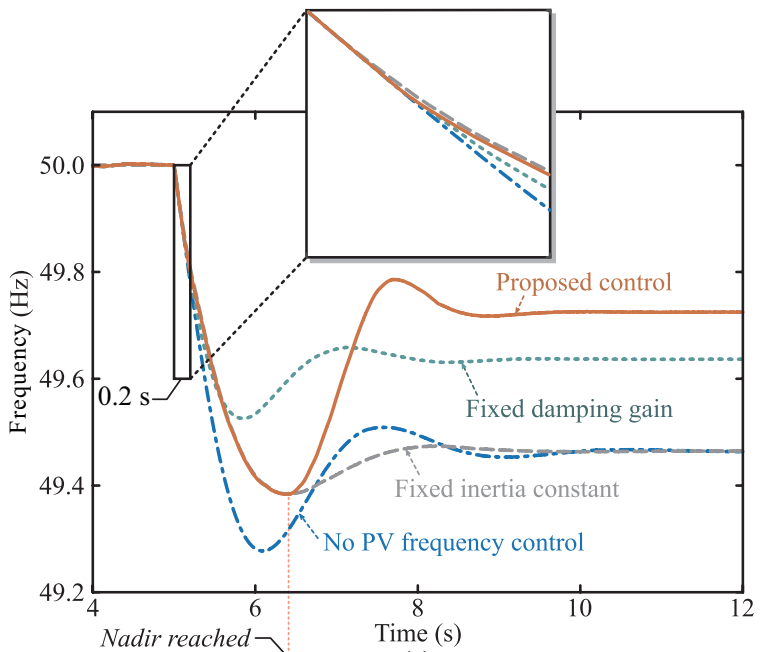

(a)

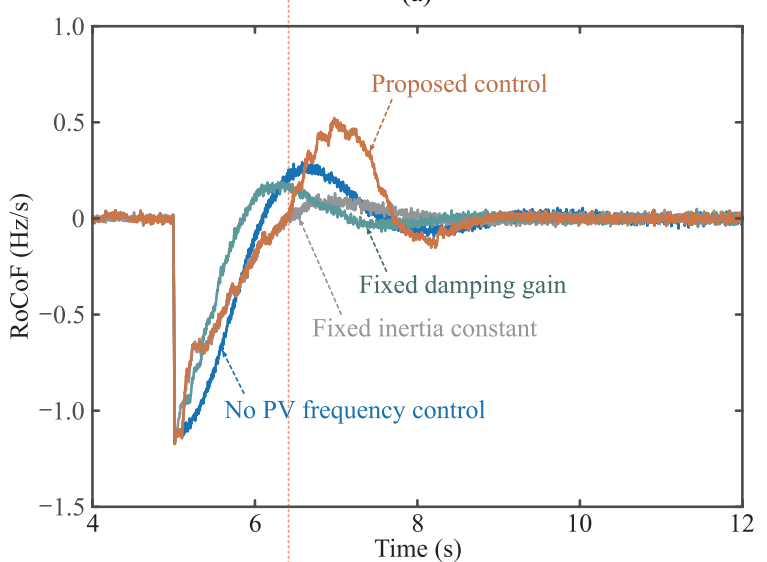

(b)

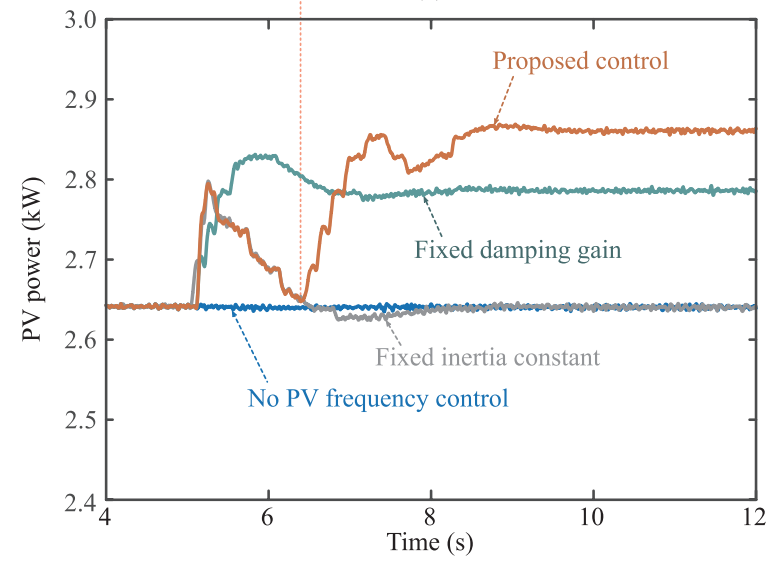

(c)

Fig. 9. System dynamics of the PV system under load step at $t=5 \mathrm{~s}$ with the proposed control and the single VIC and FDC: (a) grid frequency, (b) instantaneous RoCoF, and (c) PV output power.

to the FDC after the nadir. More importantly, the recovery frequency is even higher than the case with the fixed damping gain control. The reason is that the damping gain in the proposed control is calculated by the maximum steady-state frequency deviation, whereas the fixed damping gain is calculated by the maximum instantaneous frequency deviation.
Furthermore, it can be seen in Fig. 9(b) that the RoCoF after the nadir under the proposed control is larger than that with other control methods. This is caused by the frequency reference (the targeted recovery frequency) step due to the operation mode switching from the VIC to the FDC. However, as the change rates of the inertia constant and damping gain are well limited, the RoCoF in this stage is much smaller than the one at the early stage of the oscillation, which is acceptable by the grid code. As for the PV output power, it can be seen in Fig. 9(c) that with the fixed inertia constant control, the PV system increases the output power at the early stage of the oscillation, but returns to the initial point after the oscillation, meaning that the PV system ceases the frequency support to the grid. With the fixed damping gain control, the PV system can keep supporting the grid frequency after the oscillation.

However, due to the limitation on the damping gain, the PV system cannot utilize all the power reserve to maintain the grid frequency after the oscillation. When the proposed control is enabled, the PV system increases the output power at the early stage of the oscillation to damp the frequency nadir. After that, unlike the fixed inertia constant control, the proposed control makes the PV system increase the output power again to achieve a larger recovery frequency. Compared with the fixed damping gain control, the proposed control makes full use of the PV system power reserve to support the grid after the frequency oscillation, leading to a smaller steady-state frequency deviation, as shown in Fig. 9(a).

It can be concluded from this case study that the proposed control effectively coordinates the VIC and the FDC, based on which the deficiency of the single VIC and FDC can be avoided. Consequently, the regulation capability of the PV system is maximized and the frequency quality is improved to a large extent.

\section{B. Comparison With Advanced VIC and FDC}

The proposed control strategy is further compared with the fixed inertia constant $\&$ damping gain control and the alternating inertia constant control. Under the same conditions, the system dynamics under a $0.45 \mathrm{~kW}$ load step are shown in Fig. 10 and the control parameters are shown in Fig. 11. The system performance is discussed in the following:

- With the alternating inertia constant control, the frequency behaves similarly with respect to the one controlled by the fixed inertia constant. In contrast, the inertia constant turns to zero when the frequency goes toward the rated (see Fig. 11), making the PV system not generate any power for frequency support, as shown in Fig. 10(c). In this case, energy consumption is reduced. Moreover, like the fixed inertia constant control, the PV system does not contribute to an increased recovery frequency and the power reserve is not efficiently utilized in this regard. Notably, as shown in Fig. 10(a), when the frequency goes toward the rated after the nadir, the frequency oscillation becomes even severe by setting a relatively small inertia constant, which is in conflict with the results shown in [27]. The reason is that the method was designed to deal with the frequency issues 


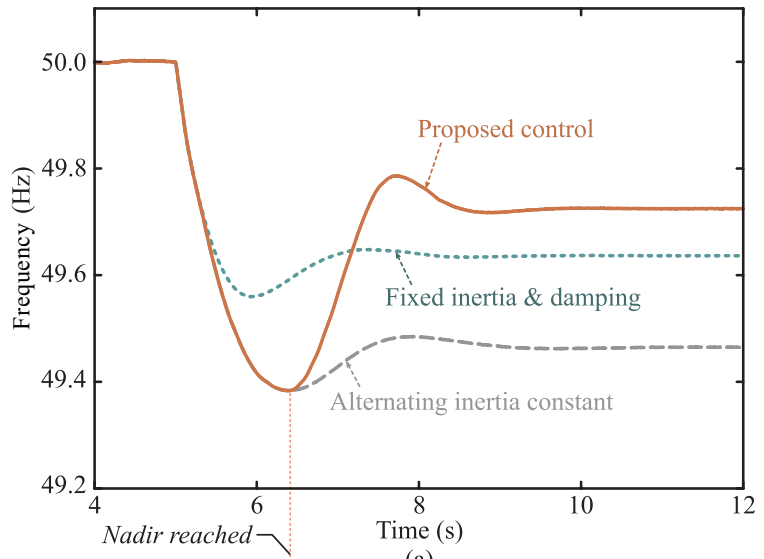

(a)

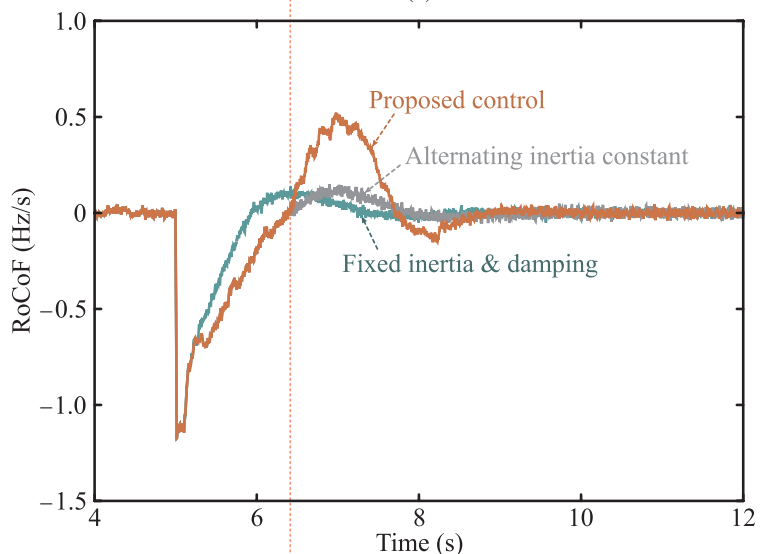

(b)

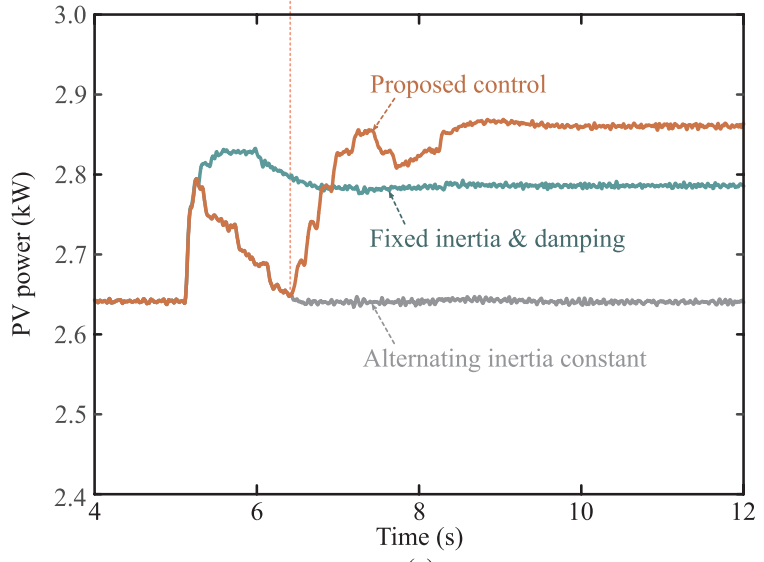

(c)

Fig. 10. System dynamics of the PV system under the load step at $t=5 \mathrm{~s}$ with the proposed control and different advanced VIC and FDC: (a) grid frequency, (b) instantaneous RoCoF, and (c) PV output power.

in strong grids, where the frequency will not reach a new steady-state after load changes. In such a case, the main objective of the strategy is to help the frequency move back to the rated. From the perspective of the new steady-state, the control strategy makes the frequency oscillate.

- When the fixed inertia constant $\&$ damping gain control is adopted, the RoCoF is decreased and the frequency nadir is increased effectively. Moreover, as the FDC is considered in this strategy, the recovery frequency is increased, i.e., the PV system continues supporting the grid frequency after

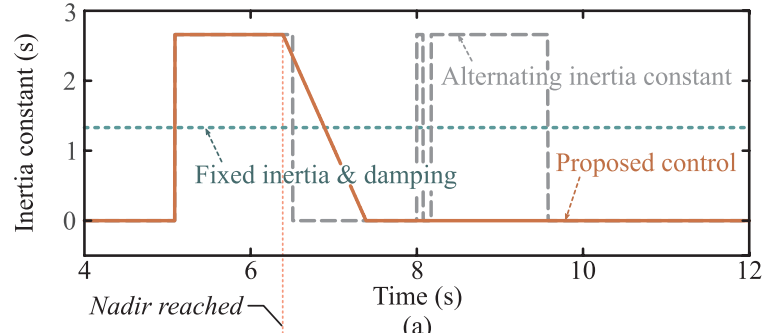

(a)

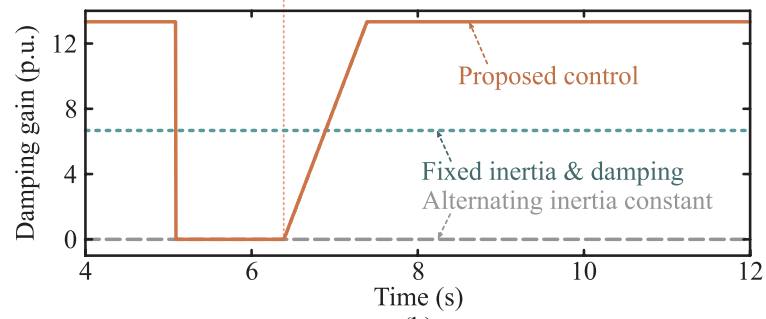

(b)

Fig. 11. Inertia constant and damping gain when the load step is applied to the PV system at $t=5 \mathrm{~s}$ with the proposed control and different advanced VIC and FDC: (a) inertia constant and (b) damping gain.

the oscillation. In summary, this strategy utilizes the power reserve of the PV system more efficiently compared to the single VIC or FDC, based on which the grid frequency quality can be improved. However, as the power reserve is evenly divided for the VIC and the FDC, only half of the power reserve is applied to support the grid frequency after the oscillation, while the rest is not utilized at all in the recovery frequency support.

- By contrast, if the proposed control is enabled, the RoCoF is decreased and the frequency nadir is increased as well. After reaching the nadir, the inertia constant gradually decreases to zero while the damping gain increases to the maximum within $1 \mathrm{~s}$, as shown in Fig. 11. As the maximum damping gain is employed after the oscillation, the recovery frequency is increases adequately. Compared with the alternating inertia constant control, the FDC is considered in this strategy, which makes the PV system increase the output power again after reaching the nadir. Compared with the fixed inertia \& damping control, the proposed control makes use of the power reserve more flexibly, by which the power reserve can be completely utilized after the oscillation. As a result, the recovery frequency under the proposed control is much higher. It should be pointed out that the maximum RoCoF during the incident with the proposed control is identical to the one with the fixed inertia \& damping control. However, when the system suffers from a severer disturbance, the proposed method will be more effective to damp the RoCoF due to the larger inertia constant at the early stage of the incident.

The frequency quality indices with different control strategies are summarized in Table V. It can be seen in Table V that the proposed control can realize comprehensive optimal frequency quality. Specifically, only the fixed damping gain and the fixed inertia constant \& damping gain control can ob- 
TABLE V

Frequency Quality Indices With DifFerent Frequency Control Strategies of PV Systems*

\begin{tabular}{lccc}
\hline \hline Control method & Nadir & $\mid$ RoCoF $\left.\right|^{* *}$ & $\begin{array}{c}\text { Recovery } \\
\text { frequency }\end{array}$ \\
\hline No PV control & $49.28 \mathrm{~Hz}$ & $1.10 \mathrm{~Hz} / \mathrm{s}$ & $49.46 \mathrm{~Hz}$ \\
Fixed inertia constant & $49.38 \mathrm{~Hz}$ & $\mathbf{0 . 9 5 ~ H z} / \mathbf{s}$ & $49.46 \mathrm{~Hz}$ \\
Fixed damping gain & $\mathbf{4 9 . 5 3 ~ H z}$ & $1.05 \mathrm{~Hz} / \mathrm{s}$ & $49.64 \mathrm{~Hz}$ \\
Fixed inertia \& damping & $\mathbf{4 9 . 5 6 ~ H z}$ & $1.00 \mathrm{~Hz} / \mathrm{s}$ & $49.64 \mathrm{~Hz}$ \\
Alternating inertia & $49.38 \mathrm{~Hz}$ & $1.00 \mathrm{~Hz} / \mathrm{s}$ & $49.46 \mathrm{~Hz}$ \\
Proposed control & $49.38 \mathrm{~Hz}$ & $1.00 \mathrm{~Hz} / \mathrm{s}$ & $49.72 \mathrm{~Hz}$ \\
\hline \hline
\end{tabular}

* The indices in bold are those better than the proposed.

** Measured in the first $0.2 \mathrm{~s}$ of the incident.

tain a larger nadir, while only the fixed inertia constant control can achieve a smaller RoCoF than the proposed control. More importantly, with the proposed control, the recovery frequency reaches the highest, indicating that the power reserve of the PV system is fully utilized.

\section{CONCLUSION}

This paper proposed a coordinated strategy of the VIC and the FDC for PV systems with power reserve. For the grid frequency quality, the RoCoF, the instantaneous frequency deviation and the steady-state frequency deviation were of importance. By properly coordinating the VIC and the FDC as proposed in this paper, the power reserve of PV systems was efficiently utilized to improve the frequency quality. In an under-frequency issue, for example, the VIC will damp the RoCoF and increase the frequency nadir (related to the instantaneous frequency deviation). Once the frequency reaches the nadir, the PV system will be switched to the FDC to increase the recovery frequency (related to the steady-state frequency deviation). Compared with the existing VIC and FDC of PV systems, the proposed control can optimally utilize the power reserve of PV systems, and in turn, the grid frequency can be improved in a cost-effective manner.

\section{REFERENCES}

[1] F. Blaabjerg, Y. Yang, D. Yang, and X. Wang, "Distributed powergeneration systems and protection," in Proceedings of the IEEE, vol. 105, no. 7, pp.1311-1331, Jul. 2017.

[2] Q. Peng, Q. Jiang, Y. Yang, T. Liu, H. Wang, and F. Blaabjerg, "On the stability of power electronics-dominated systems: Challenges and potential solutions," in IEEE Transactions on Industry Applications, vol. 55, no. 6, pp. 7657-7670, Nov. 2019.

[3] J. Fang, H. Li, Y. Tang, and F. Blaabjerg, "On the inertia of future moreelectronics power systems," in IEEE Journal of Emerging and Selected Topics in Power Electronics, vol. 7, no. 4, pp. 2130-2146, Dec. 2019.

[4] P. Kundur, N. J. Balu, and M. G. Lauby, Power System Stability and Control, New York, NY, USA: McGraw-hill, 1994.

[5] E. Tröster and E. GmbH, "New German grid codes for connecting PV systems to the medium voltage power grid," in Proceedings of 2nd International Workshop Concentrating Photovoltaic Power Plants: Optical Design, Production, Grid Connection, Darmstadt, Germany, Mar. 2009, pp. 1-4.

[6] IEEE Std 1547-2018 Revis. IEEE Std 1547-2003, IEEE Standard for Interconnection and Interoperability of Distributed Energy Resources with Associated Electric Power Systems Interfaces. IEEE, Apr. 2018.
[7] Hydro-Quebéc, "Technical requirements for the connection of generating stations to the hydro-québec transmission system," Montréal, QC, Canada, Tech. Rep. Decision D-2018-145, Jan. 2019.

[8] SolarPower Europe, "Global market outlook for solar power 2020-2024," Brussels, Belgium, Tech. Rep., Jun. 2020.

[9] E. Waffenschmidt and R. S. Hui, "Virtual inertia with PV inverters using DC-link capacitors," in Proceedings of 2016 18th European Conference on Power Electronics and Applications, Karlsruhe, Germany, Sep. 2016, pp. 1-10.

[10] P. Rodríguez, C. Citro, J. I. Candela, J. Rocabert, and A. Luna, "Flexible grid connection and islanding of SPC-based PV power converters," in IEEE Transactions on Industry Applications, vol. 54, no. 3, pp. 26902702, May 2018.

[11] C. Verdugo, A. Tarraso, J. I. Candela, J. Rocabert, and P. Rodriguez, "Centralized synchronous controller based on load angle regulation for photovoltaic power plants," in IEEE Journal of Emerging and Selected Topics in Power Electronics, 2020, early access, doi: 10.1109/ JESTPE.2020.2995339.

[12] Q. Peng, A. Sangwongwanich, Y. Yang, and F. Blaabjerg, "Grid-friendly power control for smart photovoltaic systems," in Solar Energy, vol. 210, pp. 115-127, Nov. 2020.

[13] A. F. Hoke, M. Shirazi, S. Chakraborty, E. Muljadi, and D. Maksimovic, "Rapid active power control of photovoltaic systems for grid frequency support," in IEEE Journal of Emerging and Selected Topics in Power Electronics, vol. 5, no. 3, pp. 1154-1163, Sep. 2017.

[14] A. Sangwongwanich, Y. Yang, F. Blaabjerg, and D. Sera, "Delta power control strategy for multistring grid-connected PV inverters," in IEEE Transactions on Industry Applications, vol. 53, no. 4, pp. 3862-3870, Jul. 2017.

[15] E. I. Batzelis, G. E. Kampitsis, and S. A. Papathanassiou, "Power reserves control for PV systems with real-time MPP estimation via curve fitting," in IEEE Transactions on Sustainable Energy, vol. 8, no. 3, pp. 12691280, Jul. 2017.

[16] A. Sangwongwanich, Y. Yang, and F. Blaabjerg, "A sensorless power reserve control strategy for two-stage grid-connected PV systems," in IEEE Transactions on Power Electronics, vol. 32, no. 11, pp. 8559-8569, Nov. 2017.

[17] Q. Peng, Z. Tang, Y. Yang, and F. Blaabjerg, "Event-triggering power reserve control for grid-connected PV systems," in Proceedings of 2020 IEEE Applied Power Electronics Conference and Exposition (APEC), New Orleans, LA, USA, Mar. 2020, pp. 417-423.

[18] E. I. Batzelis, G. Anagnostou, I. R. Cole, T. R. Betts, and B. C. Pal, "A state-space dynamic model for photovoltaic systems with full ancillary services support," in IEEE Transactions on Sustainable Energy, vol. 10, no. 3, pp. 1399-1409, Jul. 2019.

[19] S. I. Nanou, A. G. Papakonstantinou, and S. A. Papathanassiou, "A generic model of two-stage grid-connected PV systems with primary frequency response and inertia emulation," in Electric Power Systems Research, vol. 127, pp. 186-196, Oct. 2015.

[20] X. Huang, K. Wang, G. Li, and H. Zhang, "Virtual inertia-based control strategy of two-stage photovoltaic inverters for frequency support in islanded micro-grid," in Electronics, vol. 7, no. 11, p. 340, Nov. 2018.

[21] B. Crăciun, T. Kerekes, D. Séra, and R. Teodorescu, "Frequency support functions in large PV power plants with active power reserves," in IEEE Journal of Emerging and Selected Topics in Power Electronics, vol. 2, no. 4, pp. 849-858, Dec. 2014.

[22] J. C. Hernández, P. G. Bueno, and F. Sanchez-Sutil, "Enhanced utilityscale photovoltaic units with frequency support functions and dynamic grid support for transmission systems," in IET Renewable Power Generation, vol. 11, no. 3, pp. 361-372, 2017.

[23] J. Driesen and K. Visscher, "Virtual synchronous generators," in Proceedings of 2008 IEEE Power and Energy Society General Meeting - Conversion and Delivery of Electrical Energy in the 21st Century, Pittsburgh, PA, USA, Jul. 2008, pp. 1-3.

[24] H. Beck and R. Hesse, "Virtual synchronous machine," in Proceedings of 2007 9th International Conference on Electrical Power Quality and Utilisation, Barcelona, Spain, Oct. 2007, pp. 1-6.

[25] S. Dong and Y. C. Chen, "A method to directly compute synchronverter parameters for desired dynamic response," in IEEE Transactions on Energy Conversion, vol. 33, no. 2, pp. 814-825, Jun. 2018.

[26] B. K. Poolla, S. Bolognani, and F. Dörfler, "Optimal placement of virtual inertia in power grids," in IEEE Transactions on Automatic Control, vol. 
62, no. 12, pp. 6209-6220, Dec. 2017.

[27] J. Alipoor, Y. Miura, and T. Ise, "Power system stabilization using virtual synchronous generator with alternating moment of inertia," in IEEE Journal of Emerging and Selected Topics in Power Electronics, vol. 3, no. 2, pp. 451-458, Jun. 2015.

[28] D. Li, Q. Zhu, S. Lin, and X. Y. Bian, "A self-adaptive inertia and damping combination control of VSG to support frequency stability," in IEEE Transactions on Energy Conversion, vol. 32, no. 1, pp. 397-398, Mar. 2017

[29] F. Wang, L. Zhang, X. Feng, and H. Guo, "An adaptive control strategy for virtual synchronous generator," in IEEE Transactions on Industry Applications, vol. 54, no. 5, pp. 5124-5133, Sep. 2018.

[30] U. Markovic, Z. Chu, P. Aristidou, and G. Hug, "LQR-based adaptive virtual synchronous machine for power systems with high inverter penetration," in IEEE Transactions on Sustainable Energy, vol. 10, no. 3, pp. 1501-1512, Jul. 2019.

[31] A. S. Mir and N. Senroy, "Self-tuning neural predictive control scheme for ultrabattery to emulate a virtual synchronous machine in autonomous power systems," in IEEE Transactions on Neural Networks and Learning Systems, vol. 31, no. 1, pp. 136-147, Jan. 2020.

[32] Y. Yang, K. A. Kim, F. Blaabjerg, and A. Sangwongwanich, Advances in Grid-Connected Photovoltaic Power Conversion Systems, Woodhead Publishing, 2018

[33] X. Li, H. Wen, Y. Zhu, L. Jiang, Y. Hu, and W. Xiao, “A novel sensorless photovoltaic power reserve control with simple real-time MPP estimation," in IEEE Transactions on Power Electronics, vol. 34, no. 8, pp. 7521-7531, Aug. 2019.

[34] ENTSO-E, "Network code on load-frequency control and reserves," Brussels, Belgium, Tech. Rep., Jun. 2013.

[35] Energinet.dk, "Technical regulation 3.2.3 for thermal plants above 11 kW,” Tech. Rep. 14/26077-130, Oct. 2017.

[36] C. Broderick, "Rate of change of frequency (RoCoF) withstand capability," ENTSOE, Brussels, Belgium, Tech. Rep., Jan. 2018.

[37] B. K. Poolla, D. Groß, and F. Dörfler, "Placement and implementation of grid-forming and grid-following virtual inertia and fast frequency response," in IEEE Transactions on Power Systems, vol. 34, no. 4, pp. 3035-3046, Jul. 2019.

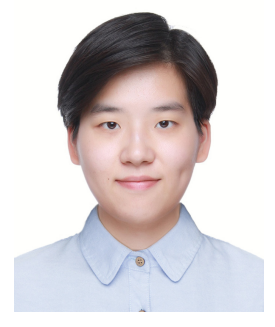

Qiao Peng received the B.Eng. degree in electrical engineering and automation from Sichuan University, Chengdu, China, in 2015. She is currently working toward the Ph.D. degree at Aalborg University, Aalborg, Denmark.

Her research interests include stability and control of power systems with renewable energy sources and power electronics, virtual inertia issues of gridconnected converters, and integration of grid-friendly photovoltaic systems.

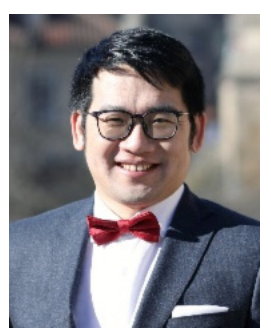

Yongheng Yang received the B.Eng. degree from Northwestern Polytechnical University, China, in 2009 and the Ph.D. degree from Aalborg University, Denmark, in 2014. He was a postgraduate student with Southeast University, China, from 2009 to 2011. In 2013, he spent three months as a Visiting Scholar at Texas A\&M University, USA. Currently, he is an Associate Professor with the Department of Energy Technology, Aalborg University, where he is also the Vice Program Leader for the research program on photovoltaic systems.

Dr. Yang is the Chair of the IEEE Denmark Section. He is now an Associate Editor for several prestigious IEEE Transactions/Journals. He is a Deputy Editor of the IET Renewable Power Generation for Solar Photovoltaic Systems. He was the recipient of the 2018 IET Renewable Power Generation Premium Award and was an Outstanding Reviewer for the IEEE Transactions on Power Electronics in 2018. His research is focused on the integration of grid-friendly photovoltaic systems with an emphasis on the power electronics converter design, control, and reliability.

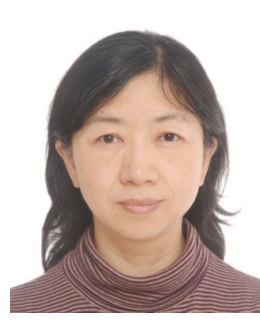

Tianqi Liu received the B.S. and the M.S. degrees from Sichuan University, Chengdu, China, in 1982 and 1986, respectively, and the Ph.D. degree from Chongqing University, Chongqing, China, in 1996, all in Electrical Engineering.

Currently, she is a professor in the College of Electrical Engineering, Sichuan University, Chengdu, China. Her main research interests are power system stability and control, HVDC, optimal generation dispatch, dynamic security analysis, dynamic state estimation and load forecasting.

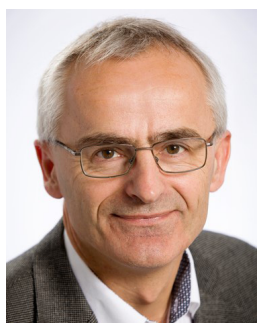

Frede Blaabjerg was with ABB-Scandia, Randers, Denmark, from 1987 to 1988. From 1988 to 1992 , he got the Ph.D. degree in Electrical Engineering at Aalborg University in 1995. He became an Assistant Professor in 1992, an Associate Professor in 1996, and a Full Professor of power electronics and drives in 1998. From 2017 he became a Villum Investigator. $\mathrm{He}$ is honoris causa at University Politehnica Timisoara (UPT), Romania and Tallinn Technical University (TTU) in Estonia.

His current research interests include power electronics and its applications such as in wind turbines, PV systems, reliability, harmonics and adjustable speed drives. He has published more than 600 journal papers in the fields of power electronics and its applications. He is the co-author of four monographs and editor of ten books in power electronics and its applications.

He has received 32 IEEE Prize Paper Awards, the IEEE PELS Distinguished Service Award in 2009, the EPE-PEMC Council Award in 2010, the IEEE William E. Newell Power Electronics Award 2014, the Villum Kann Rasmussen Research Award 2014, the Global Energy Prize in 2019 and the 2020 IEEE Edison Medal. He was the Editor-in-Chief of the IEEE Transactions on Power Electronics from 2006 to 2012. He has been Distinguished Lecturer for the IEEE Power Electronics Society from 2005 to 2007 and for the IEEE Industry Applications Society from 2010 to 2011 as well as 2017 to 2018. In 2019-2020 he serves a President of IEEE Power Electronics Society. He is Vice-President of the Danish Academy of Technical Sciences too. He is nominated in 2014-2019 by Thomson Reuters to be between the most 250 cited researchers in Engineering in the world. 